\title{
CLIMA ORGANIZACIONAL Y DESEMPEÑO LABORAL UNA REFLEXIÓN CUALITATIVA, EN LAS UNIVERSIDADES DEL DEPARTAMENTO DE LA GUAJIRA
}

\author{
MELVIS JOSEFINA ARISMENDY MORALES* \\ EVELIO JOSÉ SANTIS AGUAS**
}

\author{
Recibido 24 de Mayo de 2011/Enviado para Modif \\ icación 9 de Noviembre de 2011/Aceptado 30 de Noviembre de 2011
}

\begin{abstract}
RESUMEN
Es una reflexión para la disertación cualitativa a una investigación realizada por Arismendy (1) para la obtención del título en Magister en Gerencia Empresarial de la Universidad "Doctor Rafael Belloso Chacin" de Maracaibo en la República Bolivariana de Venezuela, acerca del enfoque de la relación existente entre el Clima Organizacional y el Desempeño Laboral en las universidades del departamento de La Guajira en Colombia, soportada desde teóricos como: Chiavenato (2), Brunet (3), Goncalves (4) Gibson y otros (5). Se plantea una situación problémica que busca incentivar la perspectiva cualitativa del mismo fenómeno estudiado, planteando premisas en las relaciones existentes entre el clima organizacional y el desempeño laboral; se propone un enfoque epistemológico desde la fenomenología de Husserl (6), con un método elegido dentro de la tradición cualitativa de la etnográfica planteado por Morse (1985) y otros, y un diseño del proceso para una nueva investigación sugerido por Bonilla y Rodríguez (7). Se plantean propósitos e interrogantes de caracteristicas cualitativas para la discusión respecto a los resultados concluyentes en el estudio realizado por Arismendy (1), los cuales pueden plantearse desde las perspectivas teóricas diversas acerca del fenómeno para encontrar la mayor significancia de datos cualitativos, de acuerdo a la oralidad de funcionarios informantes claves en el escenario de un análisis integral.

\footnotetext{
* Profesor Catedrática de la Universidad de la Guajira; Magister en Gerencia Empresarial de la Universidad "Doctor Rafael Belloso Chacin" Maracaibo 2009, Convalidación resolución 5643 del 26 de agosto de 2009 Dirección de Calidad para la Educación Superior MEN Colombia; Especialista en Gerencia Pública de la Universidad Industrial de Santander, Bucaramanga 2000; Administradora de Empresas de la Universidad de la Guajira, Riohacha 1996. Correo: arismora@hotmail.com ** Profesor Titular de la Universidad de La Guajira Colombia; Doctorado en Ciencias de la Educación Universidad “Dr. Rafael Belloso Chacin”, Maracaibo 2009, Convalidación resolución 5120 del 31 de julio de 2009 Dirección de Calidad para la Educación Superior MEN Colombia; Magister Proyectos de Desarrollo Social Universidad del Norte, Barranquilla 1994; Ingeniero Industrial Universidad de La Guajira, Riohacha 1986. Correo: esantis3@hotmail.com.
} 
Palabras claves: Clima Organizacional, Desempeño Laboral, Investigación Cualitativa e Investigación Cuantitativa.

Clasifivación JEL: D23, L25, L32.

\begin{abstract}
It is a reflection for qualitative dissertation research by Arismendy (1) to obtain the title of Master in Business Management University "Dr. Rafael Belloso Chacin" of Maracaibo in the Bolivarian Republic of Venezuela, on the approach of the relationship between organizational climate and job performance in the universities of the department of La Guajira in Colombia, supported from theoretical as Chiavenato (2), Brunet (3), Goncalves (4); $y$ Gibson and others (5). This raises a problem situation that seeks to promote the qualitative perspective of the same phenomenon studied, considering premises in the relationship between organizational climate and job performance, we propose an epistemological approach from the phenomenology of Husserl (6), using a method selected from of qualitative ethnographic tradition posed by Morse (1985) and others, and a design process for new research suggested by Bonilla and Rodriguez (7). It raises questions of purpose and quality characteristics for discussion regarding the positive results in the study by Arismendy (1), which may arise from different theoretical perspectives on the phenomenon to find the greatest significance of qualitative data, according to orality of key informants officials on stage of a comprehensive analysis.
\end{abstract}

Key words: Organizational Climate, Job Performance, and Quantitative Research Qualitative Research.

JEL Classification: D23, L25, L32.

\title{
INTRODUCCIÓN
}

La situación actual de las universidades en el Caribe colombiano, principalmente las ubicadas en el departamento de La Guajira, están sometidas a las condiciones de inestabilidad política - económica, la pérdida de valores en la sociedad, el desempleo creciente, la miseria desde el desaprovechamiento de las oportunidades de desarrollo regional, la delincuencia social producto de la falta de gobernabilidad con sentido humano, la desarticulación entre los sectores del saber y los sectores de desarrollo económico, político y social, son factores que involucran a las instituciones educativas y limita el acceso al crecimiento de la ciencia y 
la tecnología, produciendo desmotivación, falta reconocimiento y baja remuneración salarial, incidiendo en el desempeño de los funcionarios.

El estudio del Clima organizacional y desempeño laboral en las universidades del Departamento de La Guajira, realizado por Arismendy (1), determino la relación entre el clima organizacional y el desempeño laboral de los funcionarios en las instituciones de educación superior públicas y privadas en el departamento de La Guajira, dando respuesta a las relaciones y los factores externos e internos que las afecta. Pero aspectos como la efectividad y competitividad en su esencia humana deja traslucir consideraciones propias de las organizaciones que obligan a revisar la relatividad de sus resultados bañados de pragmatismo real.

En este sentido, sería importante comprender y develar el alcance que tipifica la manera de gestionar el capital humano, en este sentido se observa una atmósfera laboral entendida por encima de la aparente cuantificación positivista de datos suministrados por las circunstancias de momentos estereotipados en la descripción estadística, los cuales al ser expresados de esta manera afectan evidentemente el análisis en términos de estructura, responsabilidad, recompensa, relaciones interpersonales, metas o desafíos y cooperación; y de paso la conformación del clima humano o psicológico de toda organización y por ende el clima organizacional.

Es de recordar que, el carácter de la educación superior es un servicio público cultural con una finalidad social para todos, además de garantizar la autonomía universitaria y velar por la calidad del servicio educativo a través del ejercicio de la suprema inspección y vigilancia. Por lo tanto la educación superior deberá propender por un espíritu reflexivo, orientado al logro de la autonomía personal en quienes participan en ella, en un marco de libertad de pensamiento y de pluralismo ideológico que tenga en cuenta la universalidad de los saberes y la particularidad de las formas culturales existentes en el entorno, postulados tomados por Arismendy (1), fundamentados en la Ley 30 de 1992 de la en Colombia (8).

Al pretender reflexionar con otra opinión sobre el estudio realizado, este debería hacerse aplicando las herramientas de la tradición cualitativa, combinada con el método etnográfico en la modalidad de la teoría fenomenológica y el propósito general seria develar la relación entre el clima organizacional y el desempeño laboral para comprender el comportamiento de los funcionarios en las instituciones de educación superior del departamento de La Guajira, a partir de la experiencia y la vivencia de su función laboral. 
Una aproximación desde la perspectiva cualitativa demarca fundamentos a investigar con entradas a buscar la interpretación de la situación, desde la visión del investigador. Es posible relacionar, la exploración de la situación, seguida de la indagación sobre el enfoque epistemológico, y luego el diseño seleccionado como proceso investigativo; finalmente son las proposiciones con sus interrogantes, derivadas de la revisión de la literatura y de la observación participante las que pondrían en discusión su constructo teórico develado de la misma realidad cuestionada.

Es intentar convertirse en un punto de partida para interesados en clarificar y anticipar en nuevos hallazgos que contribuyan a consolidar el clima laboral en las universidades del departamento de La Guajira en Colombia.

\section{1. la situación problemática con respecto a la investigación}

Una aproximación desde la perspectiva cualitativa indicaría que el fenómeno en estudio busca la interpretación de la ambiente desde la visión del investigador, para ello es pertinente hacer una exploración de la situación donde se delibere las vivencias y experiencia de informantes claves a cerca de su desempeño laboral, el primer abordaje de la realidad en la que se ha de actuar se hace desde la observación simple de hechos laborales más o menos espontáneos.

En este sentido, debe revisarse la realidad mostrada y relacionada con la visión y la misión institucional en los procesos administrativos organizacionales en cada universidad, la función laboral, su desempeño percibido en los resultados alcanzados, además el ambiente académico, administrativo y financiero, el cual al parecer puede repercutir en el clima organizacional.

Entre las razones personales de reflexión se inicia con encontrar, entender y comprender argumentos por los cuales el funcionario administrativo y docenteseve afectadoen su desempeño laboral, losfactores internosy externos que impactan el clima organizacional de la universidades del departamento de La Guajira; continua con el desarrollo de métodos de tradición cualitativa, estableciendo las inferencias en sus discusiones, para identificar patrones culturales en la conceptualización inductiva de los hallazgos que facilite la comprensión e interpretación de lo relevante en la realidad cultural del administrativo y el docente en su rol de desempeño laboral; finalmente la motivación del fenómeno en estudio en su atributo humano, donde el método escogido facilite el diagnostico de vivencias laborales. 
Entre las razones prácticas, tenemos la cognición de ser del estudio como es, el comportamiento y la actitud del funcionario hacia las competencias y habilidades que se requieren en el desempeño laboral; además definir, analizar y evaluar la actividad funcional apreciada, revisada y orientada en el escenario de los factores internos y externos que pudieran afectar el clima organizacional.

Otro aspecto por definir, son las cualidades adquiridas por el funcionario administrativo y el docente en su contexto de experticia y razonamiento labor, y en su disertación cultural de disyuntiva humana comprometido con el ejercicio competitivo de su desempeño.

En función de este análisis situacional, se consideran las siguientes premisas:

a) La relación entre el clima organizacional y el desempeño laboral en las instituciones de educación superior públicas y privadas en el departamento de La Guajira están afectadas por factores internos y factores externos institucionales, sociales y culturales.

b) Factores externos impactan el clima organizacional de las Universidades del Departamento de La Guajira.

c) Factores internos impactan el clima organizacional de las Universidades del Departamento de La Guajira.

d) El desempeño laboral de los funcionarios de las Universidades del Departamento de La Guajira son afectados por factores que lo caracterizan desde sus condiciones internas y externas.

\section{Enfoque epistemológico}

Por su parte, el enfoque epistemológico se fundamenta en Husserl (6), quien argumenta que el significado central de la fenomenología subyace a la experiencia y emplaza prejuicios por estar apoyado en la intuición, la imaginación y la visión general; su énfasis es la intencionalidad de conciencia en las experiencias basada en la memoria, la imagen y el significado; en cuanto al análisis de datos, se hace a través de la metodología de la reducción con declaraciones o afirmaciones especificas y búsqueda de los significados posibles.

En este sentido Cook y Reichart (9), argumenta que la investigación cualitativa está centrada en la fenomenología y la comprensión, la cual parte de la observación naturista sin control, de manera subjetiva, con inferencias en datos, de carácter exploratoria, inductiva y descriptiva; 
interpreta fenómenos de acuerdo con los significados de las personas implicadas; el proceso y análisis de datos no se generalizable, por su condición holística con una realidad dinámica.

Por tanto, la fenomenología establece un enfoque epistémico introspectivovivencial, debido a que no presupone nada, ni el sentido común, ni el mundo natural, ni las proposiciones científicas, ni las experiencias psicológicas; se coloca antes de cualquier creencia y de todo juicio para explorar simplemente lo dado, podría considerarse como un positivismo absoluto.

\section{El Método de estudio: Etnográfico}

En cuanto al método de estudio, es el etnográfico, cuya raíz disciplinaria es la antropología; para Morse (1985), la condición holística y contextual está en su carácter reflexivo, debido a la intencionalidad de la conciencia develada en la fenomenología eidética de Husserl (6); es pretender hacer descripción comprensiva de lo realizado, expresado, asumido y concebido por el colectivo cultural de los empleados administrativos y docentes de la universidades del departamento de La Guajira, quienes involucran convergencias y divergencias sociales que construyen valores y modelos de compatibilidad para la coexistencia en medio de factores internos y externos que los puedan afectar.

Continuando con Martínez (10), quien argumenta que se debe descubrir e interpretar lo relevante de la realidad cultural, en este caso sería indagar: ¿Cuál es la cultura laboral de los funcionarios administrativos y docentes de la universidad que vendría a incidir en el clima organizacional?, la respuesta debe estaría fundamentada en la apreciación de Arnal, Justo y Latorre (1994), en el sentido de no emitir juicios de valor.

Por su parte, Goetz y LeCompte (11), indican que la descripción e interpretación de una determinada cultura se da a partir de acontecimientos particulares, lo cual indica que los acontecimientos individuales en el escenario laboral interno y externo pueden afectar el clima laboral organizacional; en este orden de ideas, Poblete (1999) agrega que el método etnográfico establece proceso y producto en un contexto de actividades empíricas y de producción.

El propósito del método etnográfico es aprender el modo de vida de una unidad social determinada, en este caso los empleados de las universidades, además construir en esquema teórico que recoja y responda a las percepciones, acciones y normas de juicio de la unidad social. 


\section{El Diseño del proceso de investigación}

En cuanto al esquema para el diseño del proceso de la investigación puede tomarse como base la propuesta de Bonilla y Rodríguez (7), el cual contiene una estructura circular compuesta por tres momentos y secciones en las cuales se establecen acciones (ver figura 1), quien considerando que la investigación cualitativa, es un proceso de "entradas múltiples", que se retroalimentan con la experiencia, el conocimiento que se va adquiriendo de la situación, y las características de simultaneidad, su circularidad puede convertirse en un diseño en espiral en la medida en que es necesario repetir un paso para avanzar, sirve al investigador como plan de trabajo, que guía sus esfuerzos para conocer la realidad estudiada.

Figura 1.

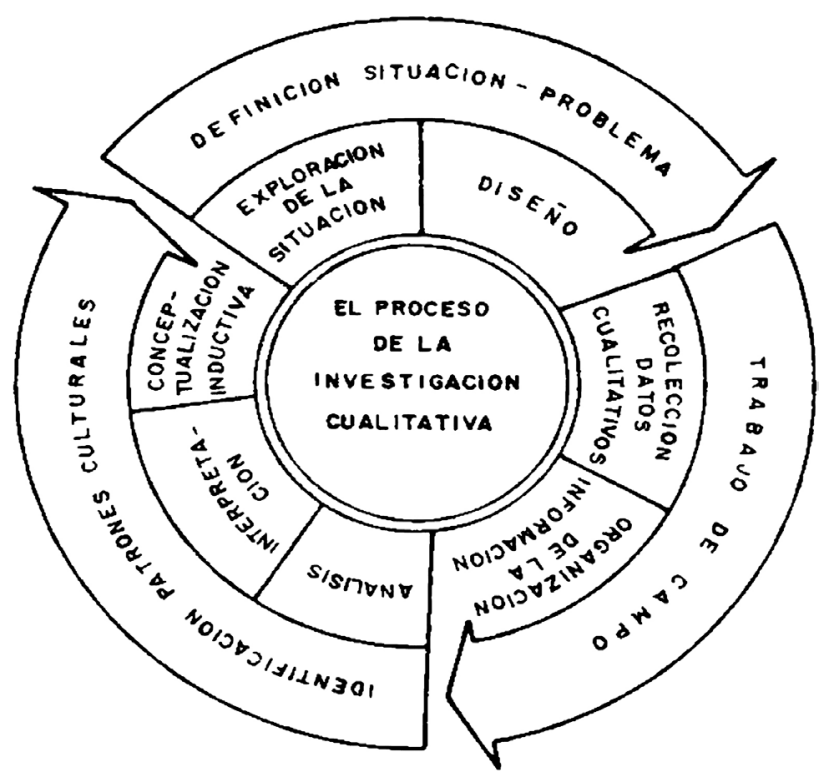

Diseño: Elsy Bonilla-Castro y Penélope Rodríguez, 1997 (7).

\section{Los Propósitos del investigador e interrogantes}

a) Comprender el comportamiento del funcionario universitario en la relación dada entre el clima organizacional y los factores que afectan el desempeño laboral en las instituciones de educación superior en el departamento de La Guajira, a partir de la experiencia y la vivencia de sus empleados. ¿Qué pasa con los funcionarios de las instituciones de educación superior en el departamento de La Guajira en la relación 
entre el clima organizacional y los factores que afectan el desempeño laboral?

b) Configurar el conocimiento apropiado del clima organizacional en su relación directa con el desempeño laboral. ¿Cuál debe ser la relación apropiada entre el clima organizacional y el desempeño laboral desde la percepción institucional y a partir de los funcionarios? ¿Es cuestión de presencia de los factores externos e internos los que impactan el clima organizacional de las Universidades del Departamento de La Guajira?

c) Develar la concepción del desempeño laboral desde la perspectiva de la vivencia de los funcionarios en las universidades. ¿Cómo saber de lo que ocultan algunos funcionarios en su desempeño laboral, en relación con sus habilidades y competencias profesionales adquiridas en su formación, pertinentes al cargo ejercido?

\section{Teorías de Entrada}

Es importante relacionar teorías que se proponen en la investigación de Arismendy (1), relacionadas con otras que posibilitan la comprensión del fenómeno, a saber:

El clima organizacional: autores como Robbin (2004), Brunet (3), Gbson (2001), Chiavenato (12), Davis (2003), y Goncalves (4) soportan fundamentos teóricos pertinentes a la dimensión de los factores externos, quienes argumentan que la vertiente organizacional que involucra la estructura y los procesos, los cuales son exclusivos de cada organización y externos al empleado; en cuanto a la dimensión de los factores internos, estos se determinan por el comportamiento organizacional del empleado y su actitud hacia el trabajo.

La actitud ante el desempeño laboral: según Alport (13), se conciben como "un estado mental y neutral de la disposición a responder a la organización a través de la experiencia y ejerce una influencia directa y/o dinámica en la conducta".

Para Byrne (14), la actitud ayuda a una persona a aumentar su auto estima, los esfuerzos para cambiarla están centrados en los beneficios de su propia imagen derivado de este cambio. En este sentido, Henao (15), plantea una disposición individual, expresadas en habilidades, destrezas y competencias, que habilitan para resolver y asumir de manera inteligente y crítica las diferencias circunstanciales de incertidumbre, racionalidad y complejidad que plantean las experiencias laborales. 
a) Las competencias y el desempeño laboral: De acuerdo con, Benavides (2002), la competencia es un comportamiento manifiestos en el desempeño laboral de una persona actuando eficazmente, la cual se soporta en el conocimiento, el deseo y la habilidad de lograr sus objetivos, razones consideradas en la producción de desempeños cualificados y en atención a las competencia genéricas, laborales o gerenciales,básicas.

En cuanto a los factores que caracterizan el desempeño laboral, autores como Schermerhorn (16), Desslerr (17), Scherman (2000), Chiavenato (18), y Maristany (19) sostienen que el grado de cumplimiento de todas las tareas que le competen a los empleados de una organización preestablecidas por la misma responsabilidad y cumplimiento de roles funcionales; adicionalmente Arismendy (1) afirma que "una cosa es que las personas vengan a trabajar y otra distinta es que se desempeñan en niveles elevados en su trabajo".

b) La innovación y la resistencia al cambio: Esto significa, que un proceso de cambio ocurre de manera eficiente si todos están comprometidos; Méndez (2002) señala que si "los cambios originan una nueva conducta en las personas, los mismos deben tener carácter de permanencia, de lo contrario se podría estar en presencia de un acto reflejo".

c) La función laboral en la Universidad: Deal y Kennedy (1985), develan a la cultura organizacional como la conducta convencional de una sociedad que comparte una serie de valores y creencias particulares y éstos a su vez influyen en todas sus acciones.

\section{El aporte de lo cuantitativo para la disertación cualitativa}

En la investigación del clima organizacional y el desempeño laboral, los aportes cuantitativos para la disertación cualitativa son:

a) Los factores externos que impactan el clima organización de las universidades, el más relevante son las condiciones físicas del lugar de trabajo, con opiniones divididas entre docentes y administrativos dependiendo del estado y las condiciones del sitio de trabajo.

b) Los factores internos que impactaron el clima organizacional de las universidades son: participación, empowerment, delegación $\mathrm{y}$, los valores. Asume una actitud de responsabilidad con logros organizacionales, toman decisiones frente a situaciones críticas, no cuenten con una autonomía frente a sus jefes, y participan en actividades grupales de capacitación; la lealtad, la responsabilidad y la honestidad son valores organizacionales comunes promovidos en las instituciones.

c) En cuanto al desempeño laboral de los funcionarios, tienen 
conocimiento de su trabajo, son organizados, se trabaja con calidad, responsabilidad y la cantidad de trabajo es acorde con el cargo; evidenciándose en los docentes y administrativos una alta presencia del desempeño en cuanto a sus funciones en las universidad.

d) Estimación muy marcada entre clima organizacional y desempeño laboral de docentes y administrativos en las universidades; se encontró una correlación $r_{s}=$ puntuación de 0,993 . Se evidenció una relación positiva muy alta y significativa entre variables; el clima organización establecido por las universidades se relaciona altamente con el desempeño laboral de los empleados.

e) En las Universidades del Departamento de La Guajira las personas categorizadas dentro de un buen clima organizacional muestran tendencias a presentar alto desempeño laboral, de igual manera quienes tengan un bajo nivel de clima organizacional presentan bajo desempeño de su trabajo.

\section{Para la discusión:}

En el escenario de las ciencias, Albert Einstein argumentaba que "no todo lo que cuenta puede ser contado, y no todo lo que puede ser contado, cuenta"; son los paradigmas los que conducen a la discusión de los fenómenos de la realidad, en un contexto, la probabilidad estadística del positivismo, la descripción de realidad y el entendimiento de la objetividad, en otro el contexto de la comprensión propositiva, las premisas y la develación subjetiva de los fenómenos, como se aprecia a continuación, en el clima organizacional y el desempeño laboral en las universidades del departamento de La Guajira:

\begin{tabular}{|c|c|c|}
\hline & a & $\mathbf{a}$ \\
\hline \multicolumn{3}{|c|}{$\begin{array}{l}\text { Realidad objeto de estu- Objetiva: determinar la incidencia Intrasubjetiva y/o Intersubjetiva: } \\
\text { dio: clima organiza- del clima organizacional en el de- comprender el comportamiento } \\
\text { cional y desempeño sempeño laboral de los funcion- de los empleados ante su fun- } \\
\text { laboral en las univer- arios. } \\
\begin{array}{ll}\text { sidades. } & \text { ción, en relación a la experiencia }\end{array}\end{array}$} \\
\hline \multicolumn{3}{|c|}{$\begin{array}{l}\text { Perspectiva: realidad Externa: cada institución concibe Interna: existen varios escenarios } \\
\text { representada en el un clima organizacional sujeto al de clima laboral de manera sub- } \\
\text { desempeño de fun- desempeñolaboral. } \\
\begin{array}{ll}\text { ciones. } & \text { los grupos culturales sociales re- } \\
& \text { lativos al desempeño laboral. }\end{array}\end{array}$} \\
\hline
\end{tabular}

Enfoque: administra- Analítico: relación entre clima Holístico: lógica inductiva partitivo funcional. organizacional y factores que cular manifestada en el empleaafectan el desempeño laboral en do que incide de manera general cada institución. en el clima organizacional de cada institución. 
Orientación hacia: la Verificación: realidad estática Descubrimiento: realidad dinámirealidad conocida o generalizable y particularista del ca natural descubierta, construdescubierta del clima desempeño laboral en el clima ida e interpretada en la mente; organizacional y el organizacional, conocida a través cambia y se devela en el mundo desempeño laboral en de la mente, no cambia, se mide social relativo de los empleados, cada universidad. en realizaciones y se sostiene.

éste incide en el clima organizacional y el desempeño laboral.

Diseño orientado al: mé- Resultado: positivistas, busca ser Proceso: naturalista fenomtodo de estudio como objetivo en la realidad del clima enológico construido desde la marco general de ref- organizacional controlado por subjetividad en el desempeño el desempeño laboral. Investi- laboral develando el clima orgación tradicional deductiva que ganizacional. Investigación inse limita a responder. ductiva etnográfica que se limita a preguntar.

Estructura: orientada a Predeterminada: el investigador Interactiva y reflexiva flexible: el la disposición de me- describe, explica y predice los investigador se aplica a la lógica tas de la investigación. fenómenos del clima organiza- inductiva percibida de las vivencional, generados por el desem- cias y experiencias de los empleapeño laboral, probado con teorías dos en su desempeño laboral, de administrativas. lo particular a lo general.

Proceso: interacción Control riguroso: el investiga- Control intersubjetivo: el investifísica entre el investi- dor esta distanciado y separado gador se aproxima, hace contacto gador y el fenómeno de los sucesos en el desempeño para revelar el clima organizaestudiado.

laboral relacionados con el clima cional, percibiendo la vivencia organizacional. del empleado, generando una riqueza interpretativa.

Procedimientos:relación Estructurados: relación de inde- Flexibles: relación de interdeentre el investigador pendencia y neutralidad que pendencia que incluye y no sepay el fenómeno estu- aprecia el clima organizacional ra al investigador conduciéndolo diado. directamente relacionado con el a descubrir el clima organizadesempeño laboral, no se afecta, cional relacionado con las experini se separan de la apreciación encias laborales del funcionario. del investigador.

Observación: contexto Controladas: el investigador hace Naturales: el investigador contexlaboral institucional medición penetrante y controla- tualiza el ambiente laboral desde da del contexto laboral en cada el empleado sin control alguno. universidad, realizando replicas y conteos.

Dato y Uso de las teor-Objetivos: inferencias más allá de Subjetivos e intersubjetivos: inferías: relación de la re- datos sólidos repetibles; la teo- encias de datos, abundantes y alidad con postulados ría administrativa organizacional profundos. Constructo teórico argumentativos. ajusta postulados en relación a administrativo es un referente las condiciones empíricas reales para el acontecer laboral del emde los empleados. pleado.

Hipótesis: conjeturas o Previas y verificables: conjeturas Emergentes y contrastables: premipremisas del fenóme- de las condiciones existentes del sas de las experiencias y vivenno en estudio. clima organizacional y el desem- cias de los empleados que reqpeño laboral que requieren ser uieren ser exploradas, inducidas confirmados, inferenciados y de- y descritas. ducidos.

Análisis: razonamiento Deductivo: el análisis del contexto Inductivo: el análisis de perspicadel contexto de la re- laboral relacionado entre las uni- cia del ambiente laboral, se detalalidad del fenómeno versidades puede ser comparado la desde las experiencias únicas, en estudio. con estudios similares, basado en natural y holística de intuición en la inducción probabilística del el proceso cíclico flexible dado en positivismo lógico usado en las la reducción de datos, la reconciencias exactas o naturales. strucción de estructuras y la comparación de casos, en el marco de las ciencias humanísticas. 
Conclusiones: deduc- Tendientes a la generalización: de- Tendientes a la particularidad: inciones e inferencias ducciones de la realidad del cli- ferencias en las premisas expuapreciadas del fenó- ma organizacional probada acer- estas sobre las vivencias y expemeno en estudio. ca de determinados conceptos riencias inducidas y descritas, las que se deducen de las conjeturas. cuales son develadas a través de la flexibilidad deducida de los informantes claves.

Resultados: $\quad$ reporte Válidos confiables: el investigador Válidos consenso intersubjetivo: el relacionado con el cli- utiliza un tono objetivo imper- investigador utiliza un tono perma organizacional y el sonal, no emotivo en la realidad sonal y emotivo en la realidad desempeño laboral medible del clima organizacional natural del clima organizacional resultante de las condiciones de afectado por el desempeño labodesempeño laboral; la gener- ral; la particularidad de sus resulalidad de sus resultados son am- tados es profunda en sus datos. plios.

\section{REFERENCIAS BIBLIOGRÁFICAS}

1. Arismendy, Melvis. Clima organizacional y desempeño laboral en las universidades del departamento de la Guajira. Tesis de grado para optar por título de magister en gerencia empresarial. Maracaibo: s.n., 2009.

2. Chiavenato, Idalberto. Gestión del talento humano. s.l.: Editorial McGraw Hill, 2000.

3. Brunet, L. El clima de trabajo en las organizaciones. s.l.: Editorial Trillas, 2000.

4. Goncalves, A. Clima organizacional. PHPARTNERS.COM/ARTICULOS. [En línea] [Citado el: 16 de Noviembre de 2011.] http://www. phpartners.com/articulos/download.asp.

5. Gibson, James, Ivancevich, John M. y Donnelly, James H. Las organizaciones, comportamiento, estructura y proceso. s.l.: Editorial Mc-Graw Hill, 2003.

6. Husserl, Edmund. Filosofía contemporánea. Ideas relativas a una fenomenología pura y a una filosofía fenomenológica. México: Universidad Nacioal Autónoma de México, 1997. 968-36-5-978-0.

7. Bonilla, Elsy y Rodríguez, Penélope. La investigación en ciencias sociales. Más allá del dilema de los métodos. Bogotá: Universidad de los Andes, 1997. 958-9057-72-1.

8. Congreso de la República de Colombia. Ley de la educación superior en Colombia. Ley 30 del 28 de diciembre de 1992, Diario oficial del Congresos de la República: Gaceta No. 40.700, 1992.

9. Cook, T. y Reichardt, C. Métodos cuantitativos y cualitativos en investigación evaluativa. Madrid: Eidtorial Morata, 1986.

10. Martínez, M. La investigación cualitativa (Síntesis conceptual). 1, Caracas - Venezuela: Universidad Nacional Mayor de San Marcos - 
Instituto de Investigaciones Psicológicas, 2004, Vol. 9.

11. Goetz, L. y Lecompte, M. Etnografía y diseño cualitativo en investigación educativa. Madrid : Editorial Morata, 1988.

12. Chiavenato, Idalberto. Administración de recursos humanos. s.l.: Editorial Mac-Graw Hill, 2004.

13. Alport, C. Tendencias desarrollistas y psicología. México: Editorial Iberoamericana, 1998.

14. Byrne, B. Psicología social. s.l.: Editorial Prentice Hall, 1999.

15. Henao, M. El papel de la investigación en la formación universitaria. Sitio Web Universia.net. [En línea] 2003. [Citado el: 23 de Agosto de 2011.] www.universia.net.co/docentes/articuloseducacionsuperior.

16. Schermerhorn, Jr. Administración. México: Editorial Limalusa, 2003.

17. Desseler, G. Administración de personal. México: Editorial Prentice Hall, 2001.

18. Chiavenato, Idalberto. Gestión del talento humano. s.l.: Editorial MacGraw Hill, 2005.

19. Maristany, J. Administración de los recursos humanos. Buenos Aires: Editorial Person Educación, 2000.

\section{BIBLIOGRAFÍA RECOMENDADA}

Bonilla - Castro, E. y Rodríguez, P. (2005). Más allá del dilema de los métodos la investigación en ciencias sociales. Bogotá D.C. Colombia: ediciones Uniandes, tercera edición grupo editorial norma.

Cohen, M. y Omery, A. (2003). Escuela de fenomenología para la investigación. En morse, j. (comps), asunto críticos en los métodos de investigación cualitativa. Colombia: Facultad de Enfermería, editorial. Universidad de Antioquia.

Constitución política de Colombia de 1991.

Ley general de educación en Colombia, ley 115 de 1994. Bogotá Colombia: serie documentos no 9 FECODE.

Robbins (2004). Comportamiento organizacional. Editorial prentice hall.

S.J. Taylor y R. Bodgan (1987). introducción a los métodos cualitativos de la investigación.

Strauss, A. y otro (1994). Base de la investigación cualitativa, técnicas y procedimientos para desarrollar la teoría fundamentada. Editorial universidad de Antioquia. Medellín Colombia: segunda edición y primera en español. 
Sherman y Bohlander (2000). Administración de los recursos humanos. México, Iberoamérica.

Taylor, S. y Bodgan, R. (1990). Introducción a los métodos cualitativos de investigación. La búsqueda de significados. México: edit. Paidos. 